\title{
A follow-up study confirms day/night enuresis cure in children by squatting-based exercises
}

\author{
(1) ANGEL GARCIA-FERNANDEZ1, (1) JORGE GARCIA ANDRADE², (1) CARLA BECKER ${ }^{3}$, (1) PETER PETROS ${ }^{4}$ \\ 1The National University of Córdoba, Córdoba, Argentina \\ ${ }^{2}$ Centro Urosalud Integral, Quito, Ecuador \\ ${ }^{3}$ Centro De Fisioterapia Uroginecologica Porto Alegre Brasil \\ ${ }^{4}$ Department of Surgery, University of New South Wales Professorial, St Vincent's Hospital, Sydney, Australia (retired)
}

\begin{abstract}
Objective: In 2018, we reported 86\% cure of day/night enuresis in a randomized controlled trial of 48 children applying three different squatting-based exercises. All children who were cured were by 4 weeks. A follow-up validation study which also aimed to assess whether singular squatting 10 times twice daily protocol was sufficient to cure day/night enuresis and whether most cures occur by 4 weeks.

Materials and Methods: The study was conducted by three centres, Argentina, Brazil, Equador. Intended protocol: 10 squats twice daily for 4 months. Eligibility criteria were daytime urine leakage plus night-time bedwetting. Exclusion criterion was refusal to sign consent forms. Assessment was by intention to treat, criterion for cure was complete dryness.

Results: When interrupted by the COVID virus, 25 cases had been assessed (ages: 6-11, one aged 17 years). Complete cure: Ecuador 5/8, Brazil 4/5, Argentina 7/12, 64\% cure, plus 4 (16\%) more than 50\% improved, with no further changes noted after 4 weeks. The study were analysed with a McNemar's test, with a null hypothesis of no treatment effect. Small numbers clouded definitive conclusions. Calculating 16/25 cured, the two-tailed $p=0.2301$; for $20 / 25$ cured or improved, $p=0.0051$.

Conclusion: The method is easily applicable, costs virtually nothing, does not require special equipment. It compares favourably with other methods such as bedwetting alarms. The results, $64 \%$ cure and $16 \%$ improvement support the $1^{\text {st }}$ study, and we believe are sufficiently encouraging to recommend adoption by parents and therapists.
\end{abstract}

Keywords: Day/night enuresis; ligaments; premature micturition; integral theory paradigm

\section{INTRODUCTION}

This study began as an attempt to validate a previous randomized controlled trial in 2018 which reported an 86\% cure of day/night enuresis in 48 children (34 females, 14 males), ${ }^{1}$ a surprising and unexpected result. The 2018 study originated from a question at a scientific meeting, "How can the Integral Theory paradigm² be applied to pediatric urology?" Patricia Skilling's squattingbased pelvic floor exercises were discussed. ${ }^{3}$ These work by strengthening the three reflex directional muscles which close the urethral tube (continence) and open it for evacuation of urine, Figure 1. Skilling reported greater than $50 \%$ improvement in a range of bladder conditions, including frequency, nocturia, urgency, urinary retention, but no cures, Table 1. 


\begin{tabular}{|l|l|}
\hline \multicolumn{2}{|l|}{ Table 1. Skilling data for premenopausal adult women } \\
\hline Fate of individual symptoms & $>50 \%$ improvement \\
\hline Condition & $57(82 \%)$ \\
\hline Stress incontinence $(n=69)$ & $33(68 \%)$ \\
\hline Urge incontinence $(n=44)$ & $10(83 \%)$ \\
\hline Frequency only $(12 \%)$ & $29(90 \%)$ \\
\hline Nocturia $(n=32)$ & $13(76 \%)$ \\
\hline Pelvic pain $(n=17)$ & $71 \mathrm{ml}$ \\
\hline Residual urine $202 \mathrm{ml}(\mathrm{n}=23)$ & \\
\hline $\mathrm{n}:$ Number &
\end{tabular}

The hypothesis for the original day/night enuresis study, was that bedwetting was an uncontrolled micturition caused by weakness or immaturity of the peripheral ligament or muscle control mechanism, ${ }^{4,5}$ and that squatting-based exercises may strengthen these to hopefully improve half the children as per Table 1. We hoped for improvement. We did not expect cure.

In the original protocol, the children were treated with 10 squats, 10 bridge exercises twice daily, and fit ball proprioception exercises during the once-a-week clinic review over a 4-month period. Though the treatment course was over a 4-month period, $41 / 48$ had been cured by the end of the first month. Assessment was by intention to treat. The criterion for cure was complete dryness. Fourteen patients had previously had medication treatment (desmopressin: 10, imipramine: 3, oxybutynin: 1), with no significant effect. Nineteen consulted with psychologists. Only some found some degree of impact on their self-esteem. There was no depression. $10 \%$ of patients had a history of urinary infections and 68\% were considered constipated. ${ }^{1}$

The cure was explained by strengthening the muscles/ligaments of the peripheral bladder control mechanism, Figure 1, thereby preventing activation of the micturition reflex. With reference to Figure 1, three directional muscle forces reflex stretch the vaginal membrane to support the stretch receptors " $\mathrm{N}$ " from below. This decreases the afferent impulses which, beyond a critical mass, overcome central control (white arrows) and activate micturition.

The aims of this study were to answer two questions:

1. Is singular squatting 10 times twice daily protocol sufficient to cure day/night enuresis?

2. Do most cures occur by four weeks?

\section{MATERIALS AND METHODS}

This report is a follow up study which aimed to assess whether squatting alone was sufficient to cure day/night enuresis. The intended protocol was 10 squats twice daily for 4 months. Recruitment for a planned study of 48 patients began in November
2019 in three different centres, the Integral Institute Centro De Fisioterapia Uroginecologica Porto Alegre Brasil. Recruitment was only from children referred to the various clinics.

Eligibility criteria were night-time bedwetting with or without daytime urine leakage. Exclusion criterion was refusal to sign consent forms. Assessment was done by intention to treat. The criterion for cure was complete dryness, as annotated and signed off by the parent.

The exercises were supervised by the parents. Monitoring was by daily parent diary and weekly review of child plus diary at the clinic. Because of coronavirus disease-19 (COVID-19), only 25 patients were able to be assessed. Consent for publication of unidentified patient data was obtained.

Ethics: Approval of the work plan by the Ethics Committee of CEIS Oulton, Instituto Oulton, affiliation, Faculty of Medical Sciences of the UNC under the supervision of the first author A.F.G. Institutional review board (IRB) approval was also obtained by the ACTA B-345 Registro Provincial De Investigacion En Salud. The trial was IRB approved (ACTA B-345) and registered. (ANZCTR) ACTRN12619000702112.

\section{RESULTS}

When interrupted by the COVID-19 virus, 25 cases had been assessed (ages: 6-11, one aged 17 years). Complete cure in Ecuador was in 5/8, in Brazil 4/5, in Argentina 7/12. This represents a 64\% cure, plus 4 children (16\%) more than $50 \%$ improved. As in the first trial, ${ }^{1}$ no further improvement in day/night enuresis was noted beyond 4 weeks.

\section{Statistical analysis}

Paired binary response data (per-patient presence/absence of a specific sign/symptom before vs after treatment) in the total study cohort were analysed with a McNemar's test, with a null hypothesis of no treatment effect.

The GraphPad Quickcalcs platform was used for this analysis.

(http://graphpad.com/quickcalcs/mcNemar1/). The sample size was small and gave discordant results depending on whether the four improved cases were included as "cured":

\section{Calculating $16 / 25$ cured.}

The two-tailed p-value equals 0.2301 . By conventional criteria, this difference is not statistically significant. The $p$-value was calculated with McNemar's test with the continuity correction. Chi-squared equals 1.440 with 1 degrees of freedom.

\section{Calculating $\mathbf{2 0 / 2 5}$ cured or improved.}

The two-tailed $p$-value equals 0.0051 . By conventional criteria, this difference is statistically significant. The $p$-value was 


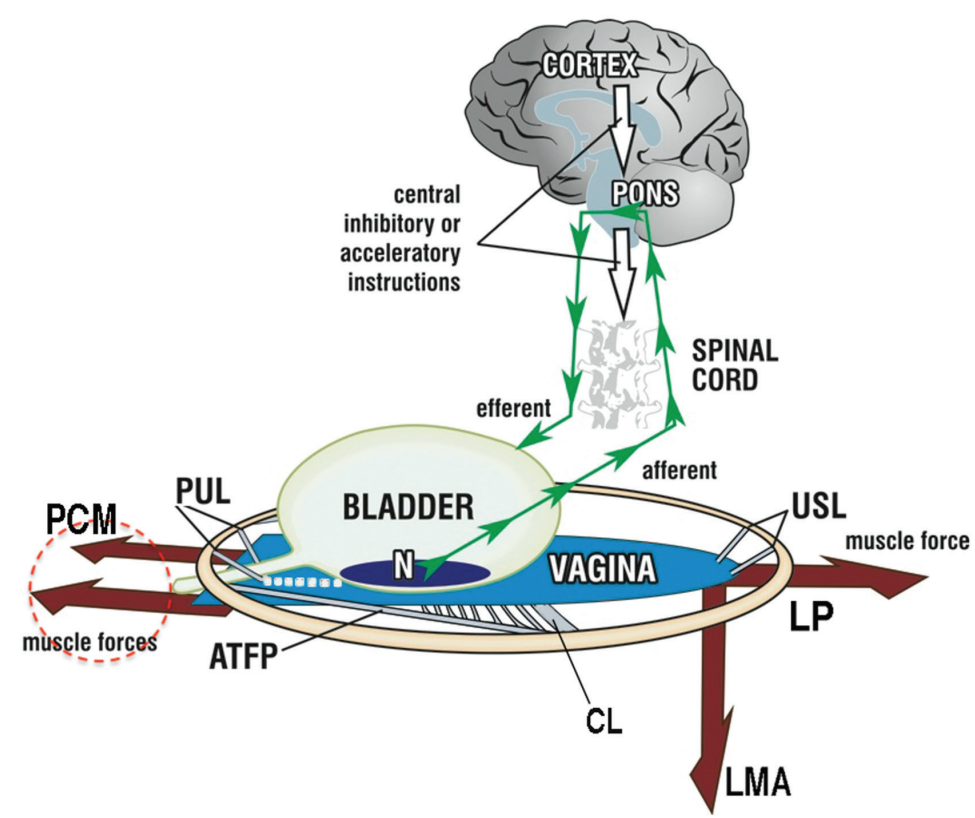

Figure 1. Control of bladder is binary Schematic 3D sagittal view. System in normal closed mode

Cortical control; afferent impulses from stretch receptors "N" are reflexly suppressed cortically (white arrows). When required, the cortex activates the micturition reflex:

Peripheral control is by a musculo-elastic mechanism which responds to cortical efferents (small arrows). Forward, backward, and downward forces (large arrows) combine to stretch the vagina tightly against its supporting ligaments, PUL (pubourethral) and USL (uterosacral), much like the membrane of a drum. The stretched vagina supports the urine column, preventing activation of the stretch receptors "N". This decreases afferent impulses to the cortex. Micturition central control (white arrows) relaxes; PCM (broken circle) relaxes; this allows the posterior muscles (arrows), LP and conjoint LMA to unrestrictedly open out the posterior wall of urethra (white broken lines) just prior to bladder evacuation by global detrusor muscle contraction.

Dysfunction (day/night enuresis). Because the striated muscle forces which activate the peripheral control mechanism contract against PUL and USL, if the muscles (large arrows) are immature, or if the ligaments are weak because of insufficient collagen deposition (again immaturity), the opposite stretching mechanism may not be able to stretch the vaginal membrane sufficiently to support the urine column pressing on "N"; "N" may fire off sufficient afferents to activate micturition during the day (urge incontinence) or at night (enuresis).

CX: Cervix; CL: Cardinal ligament; ATFP: Arcus tendineus fascia pelvis; PCM: Pubococcygeus muscle; LMA: Longitudinal muscle of the anus; LP: Levator plate

calculated with McNemar's test with the continuity correction. Chi-squared equals 7.840 with 1 degrees of freedom.

\section{DISCUSSION}

Small numbers clouded definitive conclusions and were too small for valid statistical comparison with the previous study. However, from a practical clinical perspective, 16/25 cure and
$50 \%$ improvement in four children within 4 weeks was, in our view, sufficiently positive as regards validation and, whether to recommend adoption by parents and therapists. The method is easily applicable, costs virtually nothing, does not require special equipment and in this and the other study, ${ }^{1}$ compares very favourably with other methods such as bedwetting alarms. The small number of patients and results of study did not answer the $2^{\text {nd }}$ question as to whether a singular exercise was as good as the more complex, more labour intensive three exercise regime. ${ }^{1}$ We consider day/night enuresis as an uncontrolled micturition caused by immaturity of peripheral muscle/ligament control which limits afferent impulses to cortex from bladder stretch receptors. ${ }^{1}$ We consider that what the exercises did was accelerate the maturation and cure of enuresis which comes about at puberty. Squatting exercises are collagen trophic, ${ }^{6,7}$ and we hypothesize the cure came about by strengthening the muscle/ ligament complex responsible for preventing activation of the micturition reflex. The well-known cure of enuresis at puberty fits with our hypothesis, ${ }^{1}$ of an immature peripheral control mechanism, Figure 1, strengthened by the trophic sex hormones of puberty.

We confirmed that cure, when it occurred, was universally seen by 4 weeks. This raised the question whether some children in the group who were not cured may go onto to have incontinence as adults. The $2^{\text {nd }}$ author (P.P.) has seen many such cases as adults and most seem to respond to a mid-urethral sling which works by reinforcing PUL. ${ }^{8}$

\section{CONCLUSIONS}

Though at first glance the results for taking 100\% cure as the criterion are seemingly inferior to the three-exercise protocol, ${ }^{1}$ the results from squatting only, 64\% cure and 16\% improvement are sufficiently encouraging to state that this study was confirmatory of the squatting method for cure of day/night enuresis.

\section{Contributions}

A.G.F. and P.P. conceptualised the study. A.G.F., J.G.A., C.B. arranged the three studies, collected and analysed of the data. A.G.F. and P.P. wrote and reviewed the manuscript.

\section{Ethics}

Ethics Committee Approval: Approval of the work plan by the Ethics Committee of CEIS Oulton, Instituto Oulton, affiliation, Faculty of Medical Sciences of the UNC under the supervision of the first author AFG. Institutional review board (IRB) approval was also obtained by the ACTA B-345 Registro Provincial De Investigacion En Salud. The trial was IRB approved (ACTA B-345) and registered. (ANZCTR) ACTRN12619000702112. 
Informed Consent: Consent for publication of unidentified patient data was obtained.

Peer-review: Externally peer-reviewed.

\section{DISCLOSURES}

Conflict of Interest: None for A.G.F., J.G.A., C.B. However, P.P. is the co-author of the Integral Theory.

Financial Disclosure: The authors declared that this study received no financial support.

\section{REFERENCES}

1. Garcia-Fernandez A, Petros PE. A four month squatting-based pelvic exercise regime cures day/night enuresis and bowel dysfunction in children aged 7-11 years. Cent European J Urol 2020; 73: 307-314.

2. Liedl B, Inoue $\mathrm{H}$, Sekiguchi $\mathrm{Y}$, et al. Update of the Integral Theory and System for Management of Pelvic Floor Dysfunction in Females. Eur Urol Suppl 2018; 17: 100-108.
3. Skilling PM, Petros PE. Synergistic nonsurgical management of pelvic floor dysfunction: second report. Int J Urogyne 2004; 15: 106-110.

4. Papa Petros PE. Detrusor instability and low compliance may represent different levels of disturbance in peripheral feedback control of the micturition reflex. Neurourol Urodyn 1999; 18: 81-91.

5. Petros P, Bush M. A Feedback Control System Explains Clinical and Urodynamic Bladder Instability in The Female. Pelviperineology 2016; 35: 90-93.

6. Miller BF, Hansen M, Olesen JL, et al. Tendon collagen synthesis at rest and after exercise in women. J Appl Physiol (1985) 2007; 102: 541-546.

7. Miller BF, Olesen JL, Hansen M, et al. Coordinated collagen and muscle protein synthesis in human patella tendon and quadriceps muscle after exercise. J Physiol 2005; 567: 1021-1033.

8. Ulmsten U, Petros P. Intravaginal slingplasty (IVS): an ambulatory surgical procedure for treatment of female urinary incontinence. Scand J Urol Nephrol 1995; 29: 75-82.

\section{Editorial Comment}

\section{Professor Jacob Bornstein}

Editor in Chief of Pelviperineology

The subject of this work, by Garcia-Fernandez A, Becker C and Petros PE, titled "A follow-up study confirms day/night enuresis cure in children by squatting-based exercises" is very interesting, and I believe that it should be disseminated. The study set out to validate a previous randomized controlled trial which gave an unexpected $86 \%$ cure rate for day/night enuresis. Our original view was that a larger group of patients was required to increase the substantive value of the work. We communicated this to the authors. Their reply was that there was no prospect of this work continuing possibly for some years because the countries of origin had been devastated by the COVID-19 virus. Under these circumstances, though the numbers are small, because the methodology is so simply repeated and so accessible to even the poorest of countries, we have made an editorial decision to publish it as is, so it can be challenged by other researchers.

Jacob Bornstein

editorinchief@pelviperineology.org 